\title{
Femme de conventionnel : un enjeu politique dans la république?
}

The wife of a deputy : a political issue in the republic?

Anne Jollet

\section{(2) OpenEdition \\ 12 Journals}

\section{Édition électronique}

URL : https://journals.openedition.org/ahrf/13618

DOI : $10.4000 /$ ahrf.13618

ISSN : 1952-403X

Éditeur :

Armand Colin, Société des études robespierristes

\section{Édition imprimée}

Date de publication : 1 septembre 2015

Pagination : 107-127

ISBN : 9782200929855

ISSN : 0003-4436

\section{Référence électronique}

Anne Jollet, «Femme de conventionnel : un enjeu politique dans la république? », Annales historiques de la Révolution française [En ligne], 381 | juillet-septembre 2015, mis en ligne le 01 septembre 2018, consulté le 23 avril 2022. URL : http://journals.openedition.org/ahrf/13618 ; DOI : https://doi.org/ 10.4000/ahrf.13618 


\section{FEMME DE CONVENTIONNEL : UN ENJEU POLITIQUE DANS LA RÉPUBLIQUE?}

Anne JOLLET

L'article veut interroger les fonctions des « femmes de » dans le contexte de la Révolution française et, ici, plus spécifiquement de la Convention nationale. En étudiant la place que les épouses de député occupent dans la vie politique particulièrement passionnée et conflictuelle de l'époque de la Convention, il ne s'agit pas seulement d'aborder la question des entourages des élus, mais aussi de problématiser la question de l'espace que ces épouses peuvent occuper par elles-mêmes. II s'agit de ne pas les installer d'emblée dans la dépendance, mais d'étudier comment certaines d'entre elles peuvent se saisir de l'égalité revendiquée et en cours d'acquisition de la femme mariée pour développer leur initiative politique propre. Ces actions politiques dans l'espace public de la «femme de ", que nous aborderons ici à partir de l'exemple d'Adélaïde Guéritault, épouse du conventionnel de la Vienne, François Piorry, permettent de comprendre la façon dont la lecture sociale individualiste des révolutionnaires se combine avec le poids de longue durée d'un familialisme, porteur d'interdépendance et de co-responsabilité sociale qui, pour longtemps, bridera la possible expression politique des « épouses de ».

Mots-clés : femme, épouse, Révolution française, Convention nationale, député, élu, Poitiers, Vienne, Adélaïde Guéritault, François Piorry.

Dans le contexte du colloque 1792. Entrer en république, initié par l'ANR Actapol, l'IHRF et la Société des études robespierristes pour le 
$200^{\mathrm{e}}$ anniversaire de la Première République (septembre 2012) ${ }^{1}$, l'évidence est apparue que saisir la question au niveau des conventionnels pouvait d'emblée exclure les femmes de l'entrée en république et produire - ou reproduire - l'image d'un monde masculin, d'hommes vivant entre eux dans les enceintes publiques de l'Assemblée et des clubs politiques, tels que les procès-verbaux les présentent. Le fil de cette reproduction n'a certes pas été rompu par un nouveau statut des épouses, même si le lien conjugal a été renouvelé par la forme nouvelle du mariage, devenu contrat civil dans la constitution de 1791, par l'abaissement de l'âge de la majorité, par l'absence de consentement des parents, par l'adoption du divorce le 20 septembre $1792^{2}$. La délicate question du couple en politique, celle du lien entre les mœurs privées et la vie publique, s'est pourtant posée de façon tout à fait neuve et vive pendant la Révolution française, puisque les femmes entrent elles aussi en politique ${ }^{3}$.

L'actualité française du printemps 2012 avait mis sur le devant de la scène politique la question de la place sociale de la compagne du président de la république et de l'espace d'initiative personnelle dont celle-ci disposait dans la vie politique ${ }^{4}$. La réponse, informelle, était restée décevante, se contentant de prendre acte de la nécessaire discrétion politique de cette «femme de ». Par ailleurs, depuis quelques décennies, des études, surtout menées par des historiennes, se sont obstinées à montrer l'importance des références familiales dans l'imaginaire et dans les pratiques politiques ${ }^{5}$. Ces études ont souligné que les députés, comme les élus politiques de tous les niveaux, vivent dans un monde qui n'est pas fait que des espaces de l'institution politique et que beaucoup de transactions se réalisent de fait

(1) Voir Michel Biard, Philippe Bourdin, Hervé Leuwers, Pierre SERna (dir.), 1792 . Entrer en République, Paris, Armand Colin, 2013.

(2) L'article 17 du titre II de la Constitution de 1791 dispose : « La loi ne considère le mariage que comme un contrat civique ». Pour le contenu de la loi du 20 septembre 1792 concernant le divorce, Jacques GODECHOT, Les institutions de la France sous la Révolution et l'Empire, Paris, PUF, 1985, p. 246 et $s q$.

(3) Pour un point synthétique sur la question, Jacques GUILHAUMOU, Martine LAPIED, «L'action politique des femmes pendant la Révolution française», dans Christine FAURÉ (dir.), Encyclopédie politique et historique des femmes, Paris, PUF, 1997, p. 139-168. Je remercie par ailleurs Christine Fauré pour la relecture de cet article, dont le contenu et ses limites ne sont néanmoins imputables qu'à l'auteure.

(4) Dès le 12 mai 2012, le Parisien titre : «Hollande président, une nouvelle vie aussi pour sa compagne, Valérie Trierweiler » http://www.leparisien.fr/election-presidentielle-2012/ Renouveau de l'actualité de la question à propos du tweet de soutien au socialiste dissident rochelais adressé par Valérie Trierweiller le 12 juin 2012. Le 13 juin, le «Plus » du Nouvel Observateur titre : « Hollande doit-il enfin clarifier le rôle de sa compagne ? » http://leplus.nouvelobs.com/contribution/569489. Michel, 1995.

(5) Notamment, Lynn Hunt, Le roman familial de la Révolution française, Paris, Albin 
dans d'autres cercles de sociabilitét. Par ailleurs, on sait combien, pendant que dure la Révolution, ces espaces sont réellement publics et fréquentés, notamment par les femmes qui s' y font entendre ${ }^{7}$. La vie des élus de la nation se déroule ainsi, y compris dans les mois de grandes passions politiques, au sein d'espaces multiples, croisant les liens de sociabilité. Ces cercles de sociabilité sont souvent mixtes et les compagnes, les maîtresses, les épouses jouent un rôle dans cette vie qui croise évidemment sphère publique et sphère privée, dimensions politiques et affectives. Ces configurations, bien que connues, demeurent, en dépit des recherches, le plus souvent étudiées de façon disjointe des grands débats politiques et de l'action politique des représentants du peuple.

Ainsi, alors que l'on sait l'importance des considérations des conventionnels sur le mariage, souvent on ne sait même pas si ces députés sont ou non des époux. On ne sait pas s'ils vivent à Paris en compagnie de leurs épouses. On sait peu quels liens entretiennent les épouses avec les départements, à travers famille et ami/es. Pourtant, la lecture des correspondances ou des mémoires de députés apporte le témoignage de la présence de ces «épouses de ». Ces sources disent combien la sphère politique des élus est loin d'être un monde clos, combien les débats, les analyses politiques, les conflits se prolongent au-delà des séances de l'Assemblée ou des délibérations des comités. C'est l'existence de cette sociabilité mêlant liens affectifs et politiques que cet article voudrait rappeler, avec ce que cette dimension sociale a de subi, de non maîtrisé parfois, pour l'homme politique comme pour son épouse ${ }^{8}$.

Une des dimensions du sujet est d'aborder l'espace de liberté sociale et de liberté politique dont disposent ces « épouses de »; quelles sont les contraintes spécifiques qui, dans la vision familialiste de l'époque, pèsent sur elles ? Pour répondre à ces questions, il faut certes se rappeler que ces femmes ne disposent pas toutes des mêmes atouts, ne subissent pas les mêmes contraintes, du fait des différences de génération, de charges familiales, de conditions sociales, culturelles, de capital économique et

(6) Voir notamment l'étude récente de Siân Reynolds sur le couple Roland : Siân REYNOLDS, Marriage and Revolution. Monsieur et Madame Roland, Oxford, Oxford University Press, 2012. Je remercie notre collègue Malcolm Crook de m'avoir fait connaître ce livre.

(7) Dominique GodINEAU, Citoyennes tricoteuses. Les femmes du peuple à Paris pendant la Révolution française, Aix-en-Provence, éd. Alinea, 1988.

(8) On peut reprendre à ce propos la notion « d'entourage » utilisée dans des travaux récents d'histoire politique, si ce n'est que la question est abordée ici aussi en privilégiant ceux qui, dans le vocabulaire de l'histoire politique contemporaine, appartiennent au « personnel politique ». Voir notamment, le numéro 8 de la revue Histoire @ politique. Politique, culture et société, mai-août 2009, http://www.histoire-politique.fr/. 
symbolique. Certaines sont des Parisiennes qui, à la façon de la jeune épouse de Camille Desmoulins, sont proches du domicile familial, entretiennent des relations anciennes avec fratrie et amies ${ }^{9}$. Beaucoup d'autres, comme les épouses des jeunes conventionnels de la Vienne, Ingrand et Piorry, ont quitté leur sociabilité provinciale et vivent à Paris avec leur époux député, souvent à l'hôtel, à proximité d'autres couples, tissant, plus ou moins selon les cas, des liens entre Paris et provinces, développant des sociabilités nouvelles et spécifiques pouvant mettre en cause l'engagement politique de leur époux.

Pourtant, on peut dire que « femme de » constitue encore un mauvais genre. L'histoire politique prend assez peu au sérieux les contraintes familiales - si ce n'est en termes d'héritage des « filles de »-, et l'histoire des femmes a longtemps préféré l'évocation de l'héroïsme des femmes affrontant seules, ou en rupture, la vie en société ${ }^{10}$. L'histoire des « femmes de » situe d'emblée au seuil du monde de l'histoire dite petite, de cette histoire des mœurs, restée en dépit du renouvellement de l'histoire de la vie privée et de la place faite aux affects, une histoire de pouvoirs occultes dénoncés par les contemporains et méritant, somme toute, de rester cachés - une histoire de pouvoirs « usurpés par leurs charmes » écrivait, parmi d'autres, un auteur de la fin des années $1790^{11}$. L'existence de ces épouses renvoie à la question de la «puissance des femmes », redoutable pour tout pouvoir, dont le déploiement a été bien évoqué par Michelle Perrot ${ }^{12}$. Étudier la dimension politique de l'existence de ces femmes de conventionnels ne va donc pas de soi, mais il semble désormais possible de l'introduire.

À ce stade, il s'agira de poser quelques jalons d'une histoire qui pourrait ne pas reproduire la coupure entre vie privée et vie publique et ne pas renvoyer les femmes, cette « précieuse moitié de la République » disait Rousseau (dont ces « épouses de »), à une nullité dans la sphère publique, et singulièrement la sphère politique, qui ne rend pas compte de la réalité

(9) Jean-Paul BerTAUd, Camille et Lucile Desmoulins. Un couple dans la tourmente, Paris, Presses de la Renaissance, 1986.

(10) Cependant l'histoire des femmes inclut bien sûr la vie des femmes mariées. Ainsi les études nombreuses sur les mobilisations féminines pendant la Révolution ont montré l'importance des femmes mariées, donc des « épouses de » dans ces mobilisations. Je renvoie notamment à l'étude pionnière de Dominique GODINEAU, op. cit.

(11) Jean-Jacques SÜE, Essai sur la physiognomie des corps vivans considéré depuis l'homme jusqu'à la plante, Paris, 1797, p. III-IV. Voir à ce sujet, notamment, E. LAIRTULLIER, Les femmes célèbres de 1789 à 1795 et leur influence dans la Révolution, 2 volumes, Paris, 1840.

(12) Michelle PERROT, « Pouvoir des hommes, puissance des femmes ? L'exemple du XIX ${ }^{\mathrm{e}}$ siècle », dans Michelle PERROT, Les femmes ou les silences de l'histoire, Paris, Champs Flammarion, p. 213-225. 
du moment. Nous partirons de la faible place de la problématique dans l'historiographie politique de la Révolution française, notamment dans les dictionnaires biographiques, pour mesurer ensuite les perspectives ouvertes par ce questionnement à partir d'un exemple emprunté à la vie politique de la Vienne.

\section{Vous avez dit « marié » ? L’impasse des notices biographiques}

Partons de la manière dont les notices biographiques de conventionnels sont construites. Jusque dans les dictionnaires spécialisés, les précisions concernant le statut matrimonial des députés sont le plus souvent secondaires et aléatoires. L'on sait pourtant la grande sensibilité de ceux qui pensent alors la société et son devenir à la question du mariage ; l'on sait le jugement négatif des penseurs du XVIII ${ }^{\mathrm{e}}$ siècle sur le célibat et la façon dont ce débat a été porté par les députés à travers toute la Révolution. On sait aussi les contradictions de cette valorisation puisque le mariage peut créer des dépendances néfastes, induisant des comportements masculins pervertis dont Louis XVI a fourni l'exemple, repris à satiété dans l'argumentaire politique ${ }^{13}$. Cependant, faste ou néfaste, le mariage est socialement crucial. Cette préoccupation des acteurs n'a pourtant pas été prise au sérieux par les biographes. Les députés eux-mêmes, si prompts à condamner le célibat imposé aux membres du clergé comme contraire à l'état de nature, si prompts à proposer des taxes sur les célibataires, à développer longuement l'association entre mariage et vertu, à faire du bonheur conjugal la condition d'une société heureuse, n'ont pas imposé ce critère à la qualité de la représentation, en dépit de leur point de vue familialiste dans de nombreux débats et alors même que la question des mœurs est l'un des axes constants de leur réflexion politique. Dans la continuité de cette préoccupation, on peut reprendre, pour rappeler combien la question est pensée comme éminemment publique et politique, y compris par les penseurs les plus radicaux de l'égalité, les mots bien connus de Saint-Just lui-même non marié et sans enfant - dans les Fragments des Institutions républicaines. Après avoir dit l'égalité des époux dans l'union, il pense utile de préciser : « Les époux qui n'ont point eu d'enfants pendant les sept premières années de leur union et qui n'en ont point adopté, sont séparés

(13) Un exemple parmi d'autres de ce topos, dans un discours de Barère, bien éloigné des questions conjugales. Le 14 nivôse an II, il fait au nom du Comité de salut public un rapport sur la marine de la République en Méditerranée. Pour justifier l'alliance des Ottomans, il recourt à l'absence de mariage comme gage de confiance diplomatique : «On sait au Divan que les républiques ne se marient pas, et que Vienne ne peut plus usurper la France par des femmes autrichiennes ». 
par la loi et doivent se quitter $»^{14}$. L'expression, dans une forme abrupte, traduit le sentiment partagé de l'importance cruciale de la création d'une famille et de la production d'une descendance (qui peut désormais se faire par l'adoption).

Cependant, la qualité d'homme marié, associant vertu privée et vertu publique, ne devient légalement vertu et exigence politiques pour les élus de la nation que dans les débats constitutionnels, puis dans la Constitution de l'an III. Le temps d'une possible et menaçante indifférenciation des sexes se clôt. Tandis que la loi confirme l'exclusion des femmes de l'activité politique, le fait d'être marié pour un homme devient exigible pour être membre du Conseil des Anciens et certains conventionnels proposent lors des débats sur le texte constitutionnel que cette condition soit imposée à tout représentant de la nation. Dubois-Crancé, qui défend alors cette extension du devoir d'être marié, exprime l'idée selon laquelle c'est « une plaisanterie de dire que des hommes peuvent n'avoir pas encore senti à l'âge de trente ans le besoin du mariage. Tout homme qui, à cet âge, ne sera pas en état de donner la vie à un autre, ne sera pas capable d'être législateur. La classe des célibataires est celle des égoïstes $\gg^{15}$.

Face à l'importance de la préoccupation des hommes de la Révolution pour la question du statut matrimonial et, de façon générale, des liens familiaux, le laconisme des approches biographiques sur la situation familiale peut surprendre ${ }^{16}$. Le Dictionnaire des Conventionnels, rédigé par Auguste Kuscinski au début du siècle, ne note ainsi que de façon aléatoire le fait que les élus de la Convention soient ou non mariés, aient ou non des enfants ${ }^{17}$.

Sans envisager l'ensemble des députés, prenons quelques exemples. Dans la notice consacrée à « Jean-Pierre-André Amar », député de l'Isère, pourtant connu par son engagement virulent sur la question de la place sociale des femmes et de leur nécessaire exclusion de la sphère politique dans les rudes débats de 1793, il n'est fait aucune mention de sa vie privée jusqu' aux dernières lignes de la notice. Là, l'auteur fait référence à une lettre de 1812 rédigée par l'ancien conventionnel annonçant la mort de sa femme et disant en termes particulièrement affectueux son attachement. «J'ai

(14) Cité dans Anita FAGE, « La Révolution française et la population », Population, 1953, vol. $8, \mathrm{n}^{\circ} 2$, p. 328

(15) Cité dans Ibidem, p. 333.

(16) Voir Jennifer HEUER, Anne VERJUS, « L'invention de la sphère domestique au sortir de la Révolution », $A H R F, \mathrm{n}^{\circ} 327$, janvier-mars 2002, p. 1 -28.

(17) Auguste KuscinSKI, Dictionnaire des Conventionnels, Paris, Société de l'histoire de la Révolution française, 1916, rééd. 1973. 
perdu l'amie la plus zélée, la plus chère à mon cœur, ma consolation, mon appui $[\ldots] \gg^{18}$. La notice suivante est consacrée au conventionnel Anthoine, député de la Moselle. Celle-ci mentionne cette fois l'existence d'une épouse, en précisant que le conventionnel s'est justifié de son absence à Paris, en décembre 1792, en disant «qu'il était allé à Metz pour soustraire sa femme aux persécutions de l'aristocratie et aux attaques de l'incivisme $»^{19}$. Sur les trente-deux conventionnels dont le patronyme commence par A, finalement seules quatre mentions d'épouse apparaissent, auxquelles on peut ajouter trois suggestions de l'existence d'une « famille ». Ainsi Albouys, député du Lot, est « père d'une famille assez nombreuse » ou Armonville, député de la Marne, pauvre, « économise de quoi acheter des meubles pour sa famille». Entre la trace éventuelle d'un apport de dot et la charge d'enfants, il ne reste pas grand-chose des épouses dans ces notices. Les représentants y sont, comme dans les délibérations des institutions politiques qui constituent la première source de ces biographies, des individus libres qui vont et viennent sans attache.

Pour certains conventionnels, la notoriété de l'épouse impose cependant des développements plus substantiels. Ainsi, à propos de Camille Desmoulins, Kuscinski précise d'emblée que « le 29 décembre 1790, il épousa Lucile Duplessis et les deux familles [avec celle de Danton] vécurent dans l'intimité ». Un peu plus loin, à propos de la nuit du 10 août 1792, l'auteur fait appel au témoignage de Lucile en tant qu'épouse : d' « un récit laissé par sa femme Lucile, il résulte qu'il fut absent de chez lui dans la nuit du 9 au 10 août et toute la journée du 10 et qu'il s'était exposé $»^{20}$.

D'autres dictionnaires, plus récents et d'ambitions diverses, donnent la même vision, centrée sur action et politique. Des dictionnaires aussi différents que le Dictionnaire des régicides de Jacques-Philippe Giboury, publié par les éditions Perrin en 1989, ou le Dictionnaire des membres du comité de Salut public de Bernard Gainot, publié par Tallandier en 1990, ne font ni l'un ni l'autre de place à l'existence ou aux manifestations des "épouses de $»^{21}$. Le Dictionnaire des régicides fait encore moins de place que le Dictionnaire des conventionnels à l'ancrage familial des députés. Desmoulins fait exception et, sous une formulation assez surprenante, l'auteur développe une approche familiale qui met en avant l'épouse et les

(18) Ibidem.

(19) Ibid.

(20) Ibid.

(21) Jacques-Philippe GIBOURY, Dictionnaire des régicides, 1793, Paris, Perrin, 1989 ; Bernard GaINOT, Dictionnaire des membres du comité de Salut public, préface de Michel Vovelle, Paris, Tallandier, 1990. 
obligations domestiques de Desmoulins : «Bien que très épris, l'homme politique sacrifiait sa femme à ses réunions au club des Cordeliers où il jouissait d'une grande influence après Danton et Marat»; cela lui permet d'enchaîner sur un abrupt raccourci sur le rôle du député dans les événements du temps : «Avec Danton, il prépara la journée du 10 août $1792 »^{22}$.

Les très sérieux dictionnaires dirigés par Edna Lemay - Dictionnaire des constituants, puis Dictionnaire des législateurs - invitent toujours à préciser dans la fiche de famille qui ouvre la notice, la situation matrimoniale du député, puis celle de sa postérité23. Mais ces renseignements ne sont pas toujours fournis et, quand ils le sont, restent des éléments d'état-civil, insérant l'épouse entre père, mère et enfants, la réduisant le plus souvent à une dot, ou à un rôle de « fille de ».

Ainsi du législateur, et futur conventionnel de la Vienne, PierreFrançois Piorry, sur lequel nous reviendrons dans la seconde partie; la notice énonce, après la situation sociale des parents, l'existence d'une sœur, puis d'une épouse. Cette dernière, Adélaïde Guéritault, native de Fleuré (Vienne), est épousée en 1784 et décède à Liège, le 23 mars 1837. Disséminées dans cette notice, on trouve trois mentions renvoyant ensuite à l'existence de cette épouse ${ }^{24}$. Reprenant les députés dont le nom commence par A dans ce Dictionnaire des Législateurs, un groupe de vingt-quatre députés, les notices précisent la situation matrimoniale de seize d'entre eux. Parmi ces seize, six (dont un ecclésiastique) sont célibataires, soit a minima le quart des élus. Cependant, parmi les huit conventionnels de la Vienne sur lesquels nous reviendrons, sept sont mariés. La proportion assez importante des célibataires dans les assemblées révolutionnaires est connue, mais elle est aussi considérée, in fine, comme de faible signification sociale. Pour ceux qui sont signalés comme ayant une épouse, une fois précisée parfois une date de mariage, parfois une origine sociale, l'existence de celle-ci n'est plus mentionnée dans le déroulement de la notice ${ }^{25}$.

Un nouveau dictionnaire des conventionnels est en cours d'élaboration dans le cadre d'une vaste enquête nationale coordonnée par les

(22) Jacques-Philippe GIBOURY, op. cit.

(23) Edna Hindie LEMAY (dir.), Dictionnaire des Constituants, 1789-1791, Oxford-Paris, Voltaire Fondation Universitas, 1991 ; Idem, Dictionnaire des Législateurs, 1791-1792, Ferney-Voltaire, Centre International d'étude du XVIII' siècle, 2007.

(24) Id., Dictionnaire des Législateurs, op. cit., p. 604-605. J'ai rédigé moi-même cette notice, déjà sensible à l'existence de cette femme à la forte personnalité et à la difficulté de lui faire une juste place, mais consciente que les notices du dictionnaire se devaient d'être uniformisées dans un cadre collectif, ce qui laissait peu de place aux questions matrimoniales.

(25) Id., Dictionnaire des Législateurs, op. cit., p. 1 -24. 
professeurs Biard, Bourdin et Leuwers ${ }^{26}$. Il permettra de savoir si les célibataires sont restés aussi nombreux dans une société où les incitations au mariage sont nombreuses. Peut-être sera-t-il, aussi, une occasion de s'interroger à nouveaux frais sur la place politique des «femmes de » et de les faire sortir de la petite histoire, de leur faire une place sur la scène publique.

\section{Les « femmes de » existent bien}

On peut considérer les traces de cette existence à différents niveaux. D'une part, on peut ressaisir un espace de la vie privée au sein duquel prend place la vie publique du député. À la façon dont Edna Lemay l'a esquissé dans sa Vie quotidienne des députés aux États généraux de 1789, ou plus récemment notre collègue anglaise, Siân Reynolds, à propos du couple Roland, on peut à travers les correspondances, ou avec plus de circonspection à travers les mémoires, recomposer un espace de vie des conventionnels, considérant la place des épouses et un déploiement propre de leur existence ${ }^{27}$. Anne Verjus a rappelé cette dimension politique de la vie familiale pendant la Révolution ${ }^{28}$. Concernant l'ensemble des conventionnels, l'enquête en cours permettra d'avancer sur les rapports de couple de ceux qui sont mariés, sur la façon dont leurs épouses sont plus ou moins impliquées, de leur propre chef ou pas, en politique. Des questions aussi simples que la présence ou non de ces épouses dans la capitale, la connaissance de leurs déplacements, de leur propre entourage familial et politique pourront certainement être mieux cernées.

Sans attendre ces résultats, on saisit à travers les bribes de correspondance, notations disparates, l'importance du choix du lieu de résidence dans l'espace parisien, choix qui s'accompagne d'éloignement ou de proximité plus ou moins acceptés. Nombre de députés partagent du fait des interconnaissances les mêmes hôtels ou trouvent des appartements dans des immeubles proches, dans un espace lui-même réduit, non loin de l'Assemblée nationale.

On a vu que Camille et Lucile Desmoulins s'installent à proximité de la famille de Danton : «Les époux Desmoulins s'installent, dans cette

(26) ACTAPOL, Acteurs et action politique en Révolution : les conventionnels, Agence Nationale de la Recherche, programme blanc, 2010.

(27) Edna Hindie LEMAY, La vie quotidienne des députés aux États généraux de 1789, Paris, Hachette, 1987 ; Siân REYNOLDS, op. cit.

(28) Anne VERJUS, Le bon mari : une histoire politique des hommes et des femmes à l'époque révolutionnaire, Paris, Fayard, 2010. 
maison de la cour du Commerce où Danton habite $»^{29}$. Le couple Roland s'installe à l'hôtel Britannique, rue Guénégaud, tout près du Pont-Neuf, et Madame Roland évoque dans une lettre qu'ils espèrent être près de Delandine et de sa femme ${ }^{30}$.

Le couple du député poitevin Piorry est installé à Paris dès l'élection à la Législative, le 13 septembre 1791. Il s'installe à « l'Hôtel de Picardie, vis-à-vis du Palais-Royal », non loin de beaucoup d'autres députés, dont Pétion, qui loge rue du faubourg Saint-Honoré, ou de Brissot, qui habite rue Guétry. Le couple Piorry vit dans le même hôtel qu'un autre couple de député de la Vienne, le couple Ingrand. Les deux députés sont jeunes et de même âge : Piorry est né en 1758 et Ingrand en 1757. Ils sont mariés depuis quelques années seulement, le premier depuis 1784 et le second depuis 1787. L'un et l'autre sont alors sans enfant (ils demeureront l'un et l'autre sans descendance). Après leur réélection à la Convention, les deux couples continuent à demeurer dans le même hôtel, 58, rue Saint-Thomas du Louvre.

Les épouses des députés s'invitent à dîner, sortent au théâtre, à l'opéra, fréquentent les tribunes des assemblées, ont une sociabilité propre qui n'est pas seulement le décalque de celle des époux, mais qui est aussi celle de leurs époux. Dans son ouvrage, Siân Reynolds rappelle combien le Paris politique de la Révolution est un petit monde dans lequel les distances entre les domiciles et les espaces politiques publics se font à pied $^{31}$. À l'intérieur de cet espace, les épouses ont elles aussi leur géographie politique, comme elles ont, en 1792, un « passé », une « culture » politique, qui les rapproche plus ou moins des tribunes de l'Assemblée, du club des Jacobins ou d'autres espaces de sociabilité politique.

Comme dans les correspondances des époux Roland, les épouses et leurs fréquentations apparaissent constamment dans les lettres des deux députés poitevins. Ces deux élus sont localement ceux qui, parmi les conventionnels, ont eu la plus grande influence sur la vie politique locale, à Poitiers, et dans le département de la Vienne, par leurs échanges de courriers, par leurs envois d'imprimés, de journaux, par leurs réseaux d'amis, de parents. Leur notoriété est restée grande et sombre au cours du XIX ${ }^{\mathrm{e}}$ siècle, entretenue par les mémoires de leurs ennemis politiques

(29) Jules Claretie, Camille Desmoulins, Lucile Desmoulins, études sur les dantonistes d'après des documents nouveaux et inédits, Paris, Plon, 1875.

(30) Siân REYNOLDS, op. cit., p. 134.

(31) Ibidem. 
poitevins ${ }^{32}$. Omniprésents dans la vie politique locale, ils ont été l'objet de travaux de recherche sur la Révolution dans la Vienne depuis plusieurs décennies ${ }^{33}$. Les deux jeunes députés correspondent très régulièrement entre eux du fait de l'envoi de l'un ou de l'autre en mission. Ils sont liés par l'amitié et par des engagements politiques proches, une fréquentation assidue des Jacobins, des votes similaires dans les grands débats de la Convention, notamment lors du jugement du roi. Ces correspondances sont éclatées en plusieurs sites de conservation, principalement les archives départementales de la Vienne et la Médiathèque de Poitiers. Les lettres conservées à la Médiathèque sont elles-mêmes dans deux fonds, le fonds des manuscrits et celui des archives révolutionnaires ${ }^{34}$. Une partie des correspondances de ces députés, qui ont été poursuivis comme «terroristes » en l'an III et contraints à ne pas revenir à Poitiers, a été détruite. Les correspondances des épouses n'ont pas été retrouvées jusqu'à aujourd'hui ${ }^{35}$.

Les lettres d'Ingrand à Piorry, pendant la mission de ce dernier dans la Vienne au printemps 1793, comportent souvent quelques mots rappelant l'existence des épouses. Dans une lettre non datée, vraisemblablement d'avril 1793, dans laquelle Ingrand évoque « l'affaire de Marat» et « la déroute de nos armées contre les révoltés », donc les grandes affaires du moment, il termine son propos par un paragraphe de considérations familiales : "Votre épouse jouit d'une assez bonne santé, elle vous écrit par ce courrier ; ma femme vous dit les choses les plus honnêtes $»^{36}$. Dans une autre lettre d'avril 1793, Ingrand écrit à Piorry, toujours dans la Vienne : « Votre épouse et la mienne font triste mine au milieu de tous ces événements ; elles seraient infiniment mieux auprès de leur ménage dans notre département ».

Quand, par la suite, c'est Ingrand qui est longuement en mission dans l'ouest, dont la Vienne, Piorry, qui est resté à la Convention, reçoit

(32) Voir en particulier Antoine-René Hyacinthe THIBAUDEAU, Souvenirs des principaux événements de la Révolution principalement dans le département de la Vienne, Poitiers, Mémoires de la Société des Antiquaires de l'Ouest, 2e série, t. XVIII, 1895 ; Antoine-Claire THIBAUDEAU, Mémoires sur la Convention et le Directoire, Paris, Éditions S.P.M., 2007.

(33) Henri CARRÉ, « Les déceptions d'un représentant en mission après le 9 thermidor (1794$1795)$ ), Poitiers, Bulletin de la Société des antiquaires de l'Ouest, 1936 ; Catherine-Louise ALLEN, François-Pierre Ingrand. Représentant en mission dans la Vienne brumaire-floréal an II, Mémoire de maîtrise d'histoire moderne, Université de Poitiers, 1993 ; Pierre MAssé, Pierre-François Piorry, conventionnel et magistrat (1758-1847), Poitiers, Mémoire de la Société des Antiquaires de l'Ouest, 4e série, tome IX, 1965.

(34) Robert FAVREAU (dir.), Inventaire sommaire des archives révolutionnaires de la ville de Poitiers, Poitiers, 1967.

(35) Ibidem.

(36) AM Poitiers, Archives révolutionnaires, série B 6, casier 113/3, Lettres d'Ingrand à Piorry. 
les courriers envoyés de Poitiers. Presque toutes les lettres se terminent par un salut à l'épouse de Piorry et un salut de l'épouse d'Ingrand, visiblement revenue dans la Vienne. Dans cet échange, on notera le passage au tutoiement entre les deux députés. Ainsi en fin d'une lettre du 2 frimaire de l'an II (22 novembre 1793), lit-on en post-scriptum : « Mes compliments à ton épouse. Fais passer je te prie sous le contreseing de la Convention la lettre ci-incluse à son adresse ». Dans une lettre du 7 frimaire, le député conclut en écrivant : « Ma femme te dit ainsi qu'à ton épouse les choses les plus honnêtes. Fais lui agréer les choses les plus affectueuses de ma part $»^{37}$. Un post-scriptum évoque une autre approche des questions familiales et matrimoniales à travers l'évocation du mariage d'un cousin de Piorry, anciennement prêtre. Ingrand y donne son point de vue sur la mariée face aux réserves du député Piorry (réserves que l'on peut supposer à travers l'échange) : «Elle est jolie et bien élevée et je le crois propre à donner de beaux enfants à la république $\gg^{38}$.

Concernant l'évocation des épouses, on note le passage du registre des « choses honnêtes » aux « choses affectueuses » puis, dans les lettres suivantes, de la part de l'épouse d'Ingrand : « Ma femme vous dit à l'un et à l'autre les choses les plus fraternelles ${ }^{39}$. Souvent suit un post-scriptum précisant qu'un « paquet» doit passer sous le contreseing de la Convention à ladite épouse Piorry. On aimerait en savoir plus sur le contenu de ces paquets. Mais ils attestent bien l'existence d'un espace spécifique de cette épouse qui reçoit un courrier régulier de Poitiers.

La correspondance garde également des traces de vie quotidienne. Dans une des premières lettres envoyées après une maladie, en pluviôse an II, Ingrand écrit à son ami : ma « femme qui s'est donnée beaucoup de peine pendant ma maladie vient de tomber malade à son tour et je crains bien qu'elle n'en soit pas aussitôt quitte que moi ${ }^{40}$. L'épouse d'Ingrand se remettant de sa maladie, le député finit une lettre le 3 ventôse an II (21 février 1794) sur : "Ma femme est mieux elle offre mille amitié à ton épouse $»^{41}$. Le 8 fructidor ( 25 août), de Fontenay-le-Peuple, il évoque ses difficultés et écrit à Piorry : « Je mettrai cette lettre sous l'adresse de ton

(37) Ibidem.

(38) Ibid., lettre du 7 frimaire an II. La question de ce mariage qui va être annoncé à la Convention préoccupe visiblement beaucoup Piorry, puisque Ingrand l'informe régulièrement sur le sujet. Lettre du 2 nivôse an II : « Ton cousin est enchanté de son épouse et je les crois fait l'un pour l'autre pour concourir utilement à leur bonheur mutuel ».

(39) Ibid., lettres d'Ingrand à Piorry, lettre du 14 frimaire an II.

(40) Ibid., lettre du 24 pluviôse an II.

(41) Ibid., lettre du 3 ventôse an II. 
épouse à qui je te prie de faire agréer les choses les plus fraternelles afin qu'elle puisse parvenir plus sûrement ».

La politique, cependant, affleure par moments et les lettres laissent entrevoir la façon dont les épouses sont impliquées par l'opinion comme par les responsables politiques dans les engagements de leurs époux. Olympe de Gouges a exprimé avec force la situation paradoxale créée par l'éviction des femmes de la scène politique active et le fait que les femmes montent à l'échafaud pour raisons politiques. La question de l'inculpation de la reine et de nombreuses « épouses de » a légitimement conduit les historien/nes à s'interroger sur la façon dont est perçue socialement la responsabilité politique de l'épouse. Comme le révèlent, de façon certes marginale, les mentions des épouses dans les conflits politiques, celles-ci se trouvent de fait considérées comme impliquées par le comportement de leurs époux, mais aussi comme impliquant leurs époux.

On trouve ainsi mention de telles situations dans quelques notices biographiques évoquées ci-dessus. Nous avons cité l'explication donnée par le député de la Moselle, Anthoine, à son absence de l'Assemblée en décembre 1792, disant «qu'il était allé à Metz pour soustraire sa femme aux persécutions de l'aristocratie et aux attaques de l'incivisme $»^{42}$. Piorry, mis en cause par Boudin, député des Ardennes, qui l'accusait d'avoir sonné le tocsin la nuit du $1^{\mathrm{er}}$ prairial an III (20 mai 1795), se justifia en démontrant son absence de Paris pour porter secours à son épouse à Poitiers, qui était «traînée dans la boue par la foule comme épouse de député ». L'administration centrale du département de la Vienne était alors aux mains des thermidoriens, des proches du conventionnel Thibaudeau avec qui Piorry et son épouse avaient été en conflit en l'an II. La période du gouvernement révolutionnaire a en effet été marquée, à Poitiers, par de forts affrontements politiques et un affrontement socio-politique entre ces deux conventionnels et leurs réseaux locaux. Antoine-Claire Thibaudeau est fils d'un constituant, avocat parmi les plus renommés de Poitiers. PierreFrançois Piorry, avocat lui aussi, est de situation sociale beaucoup plus modeste. Il a épousé une jeune orpheline, pauvre, contre l'avis de son père tandis que Thibaudeau, le plus jeune des députés de la Vienne, a épousé Mathilde Tribert, la fille d'un riche marchand de grains de Poitiers, cible de la colère populaire dès 1789 et accusé d'agiotage en l'an II $^{43}$.

(42) Auguste KUSCINSKI, op. cit.

(43) Henri CARRÉ, « Les aventures d'un grand marchand de blé en Poitou, Jérémie Tribert, 1789-1794 », Bulletin de la Société des Antiquaires de l'Ouest, $3^{\mathrm{e}}$ série, tome 11, 1937, p. 514-529. 
L'affaire qui nous intéresse ici se noue autour de l'épouse de Piorry. Celle-ci, Adélaïde Guéritault, demeure à Paris pendant toute la durée des mandats de son époux mais, comme on l'a aperçu à travers la correspondance d'Ingrand avec Piorry, elle entretient des relations épistolaires régulières avec des Poitevins ${ }^{44}$. Son frère, Jude Guéritault, fait partie des proches de Piorry et sera arrêté au moment où Piorry perdra son influence politique locale, dès le début de l'an $\mathrm{III}^{45}$. Le comportement de cette épouse traduit une grande autonomie vis-à-vis de tout son groupe familial, comme vis-à-vis de son mari. Elle semble mettre en œuvre un usage tout personnel des réseaux d'interconnaissances, réseaux liés à la fois à l'origine poitevine et au devenir national d'elle-même, de son mari et de leurs proches. Dans l'affaire évoquée ici, elle n'apparaît en contact qu'avec des hommes, avec lesquels elle négocie, échange des écrits et des propos qui, selon son propre témoignage, semblent d'un grand naturel. L'affaire est connue car elle a été utilisée contre le député Piorry à plusieurs reprises : elle a notamment été développée par le conventionnel Thibaudeau qui, après avoir été marginalisé à Poitiers pendant l'an II, triomphe après Thermidor. Ce travail est aussi l'œuvre du représentant en mission Chauvin, qui remplace les montagnards.

De quoi s'agit-il ? D'une suspicion d'agiotage, d'implication dans des affaires commerciales pas très recommandables en ces temps de réquisitions et de pénurie, de la part d'une épouse qui dispose d'un peu d'argent et est très liée par ses origines familiales aux milieux commerçants de Poitiers. En mars 1793, elle propose une affaire à Bernazais, un jeune patriote de la Vienne, alors à l'état-major de Dumouriez en Hollande : elle fournirait des fonds et Bernazais enverrait des marchandises de Hollande. Elle fait sa proposition par une lettre, qui est interceptée par Gabriel Thibaudeau, fils du constituant, alors qu'il a été placé chez les Piorry par son père. En rupture avec le conventionnel Piorry, Gabriel Thibaudeau aurait menacé de faire chanter son hôte en dévoilant la lettre s'il n'obtenait un poste de directeur des messageries à Poitiers. Cette explication est donnée par Piorry et son épouse. Les Thibaudeau rétorquent, quant à eux, que la lettre n'est pas arrivée à son destinataire et est revenue au comité des Finances, sous le timbre duquel elle avait été expédiée ; le contenu en aurait 
alors été dévoilé après que le comité eut fait appel à Gabriel Thibaudeau, comme proche du couple Piorry ${ }^{46}$.

L'incident est d'autant plus grave que Bernazais vient d'être arrêté comme suspect de complicité dans la trahison de Dumouriez et que, par ailleurs, Piorry a été nommé en septembre 1793 au comité de Surveillance des marchés, dont les neuf membres sont chargés de lutter contre les abus des fournisseurs et doivent faire preuve d'une parfaite probité. Le 30 septembre, Piorry monte à la tribune de l'Assemblée. Sans nier la démarche de son épouse, il prend la défense de celle-ci :

« J'observe que le prétendu commerce de mon épouse se réduisait à une somme de 300 livres qu'elle avait prêtée au jeune volontaire de l'Armée de la Belgique pour faire ce voyage, et que, pour se procurer ensuite la rentrée de ses fonds de toute manière que ce fut, elle demandait en échange des toiles ou tout autre marchandise du pays $»^{47}$.

Il propose ensuite sa démission du comité des marchés. Ses collègues, Gossuin et Chabot, se portent garants de sa probité. La Convention refuse sa démission et le nomme secrétaire. La lettre est envoyée au Comité de sûreté générale. De fait, lors de sa mission, Piorry surveille de près le trafic des chevaux dans la Vienne, et notamment Enenon, médecin à Poitiers, qui a été une connaissance du ménage Piorry avant son départ à Paris ; il vend des mulets pour les ambulances des armées des Alpes, à des prix beaucoup trop élevés. Son contrat a été dénoncé par Thibaudeau et par un autre conventionnel de la Vienne, Creuzé Pascal.

Reste que la liberté d'initiative de Madame Piorry a été réelle ; ses correspondances nombreuses sont avérées, comme ses rencontres avec des Poitevins à Paris. Restée dans la capitale pendant la mission de son époux, elle a notamment été sollicitée par Enenon ; ce dernier se rend à Paris pour la rencontrer, lui proposant d'obtenir la neutralité du député et de mettre elle-même un peu d'argent dans l'affaire. Piorry fera surveiller spécialement Enenon qui continue à solliciter le couple avec une incroyable impudence, en envoyant des provisions de bouche à Paris - provisions que Piorry refuse.

À travers ces épisodes, on saisit combien la fonction d'épouse de député expose. Cette place donne, de façon informelle, des moyens

(46) Ibid., p. 5. Thibaudeau père avait confié à Piorry, désormais député, son fils aîné Gabriel afin qu'il lui trouve une place à Paris et celui-ci vivait au même endroit que le député et son épouse.

(47) Archives parlementaires, t. 75, p. 359. 
d'intervention, elle crée aussi des contraintes, tant toutes les initiatives de l'épouse prennent un sens politique fort et mettent en cause le député luimême. Elle oblige aussi l'épouse à se justifier ; ainsi la femme Piorry publie une brochure justificative, le 5 octobre $1793^{48}$. Elle n'est certainement pas la seule à rédiger ce texte car sa pratique de l'écrit n'était pas très sûre. Mais en l'occurrence, retenons avant tout qu'elle rend sa parole publique et s'exprime comme épouse de député ; l'en-tête de la lettre indique en effet qu'elle émane d' « Adélaïde Gueritault », désignée comme l' « épouse du sans-culotte Piorry, représentant du peuple ». La brochure prend ainsi la forme d'une lettre qu'elle adresse à «Thibaudeau, se disant directeur des messageries, à Poitiers ». La publication d'adresses publiques par des femmes n'est à l'époque ni rare, ni surprenante, même si l'enquête sur ces pratiques reste à mener.

Dès le début de cette publication, l'épouse Piorry affirme son engagement de républicaine, épouse de sans-culotte. Cette proclamation est amplifiée par l'épigraphe choisie, qui explicite son engagement politique en nom propre : «Le sang républicain circule dans mes veines ». Cette femme d'une trentaine d'années s'adresse au jeune Thibaudeau, sensiblement de son âge, en le tutoyant et en l'interpellant de façon très directe : "Tu es, dis-tu, muni d'une lettre de moi à l'adresse de Bernazais... ». Elle demande que la lettre soit apportée au Comité de sûreté générale et elle espère " $\mathrm{y}$ démontrer [à tes juges] que non seulement tu es un aristocrate, mais encore un homme indigne de la confiance publique ». Elle fait ensuite la liste des faits pour lesquels elle est inquiétée, en prenant à témoin l'opinion : «Tu m'accuses d'avoir voulu négocier sur le sucre, le café et les toiles de Hollande », « tu m'accuses non pas d'être entrée dans le marché des mulets d'Enenon, mais d'y avoir voulu rentrer », « tu m'accuses enfin d'être de part avec un parent à qui j'ai fait avoir une commission du ministre, pour un achat de chevaux ».

Après avoir rappelé la justification victorieuse de son mari à la Convention, qui «s'est levée tout entière pour lui rendre justice», elle reconnaît la réalité de la correspondance avec Bernazais ainsi que celle de la transaction économique. Elle écrit : «Bernazais m'écrivit au mois de mars dernier que les Belges ne voulant nos assignats qu'avec beaucoup de perte, il ne pouvait exécuter mes achats qu'avec une indemnité en argent, prise sur ses propres appointements ». Elle ajoute : «Que trouves-tu donc de blâmable dans cette lettre? Tu ne peux m'accuser d'accaparement ; tu 
pourrais dire tout au plus qu'au lieu de faire venir des toiles, je songeais alors à faire venir du sucre et du café. Eh bien, n'en avais-je pas le droit et la liberté ?» La républicaine affirme fermement sa liberté à entreprendre dans le domaine économique. La question de la lutte contre l'agiotage et les accapareurs est à l'ordre du jour et Adélaïde Guéritault distingue d'emblée commerce et accaparement ${ }^{49}$. Dans la phrase suivante, l'épouse du sans-culotte Piorry se demande : «Le titre d'épouse de député doit-il m'enchaîner au point de ne pouvoir même pas tirer parti de la plus petite somme ? Consultez donc un peu sur ce fait, les hommes de bonne foi !» Le cœur de la tension est ainsi bien visible : est-il admissible que le statut d'épouse de député soit une entrave à la liberté, notamment dans le domaine économique ? Certes, elle est amenée à minimiser l'enjeu économique de ses initiatives. Elle précise en note que sa « correspondance déposée au comité de Sûreté générale prouvera que tout mon prétendu négoce ne consistait que dans la rentrée de mes cent écus ».

Face à l'accusation d' « avoir voulu entrer dans le marché d'Enenon », l'épouse Piorry reconnaît une nouvelle fois les faits : «Je conviens, écritelle - rendant publiques les allégations en même temps que sa défense -, que dans les premiers jours de mai, Enenon, chirurgien, m'a proposé d'entrer dans son marché, sous la condition que je lui ferai prêter des fonds ; mais comme je ne connaissais personne, et que d'ailleurs mon mari, alors en commission à Poitiers, se serait élevé avec juste raison contre un pareil négoce, aucune espèce de convention n'a eu lieu. Enenon s'est enfui et j'atteste que je ne l'ai pas vu depuis le mois de mai dernier $»^{50}$. Elle reconnaît la sollicitation ainsi que la position d'épouse, mais on mesure à l'énoncé des faits que son initiative personnelle est entière.

Dans la suite de sa défense, on retrouve la tension entre les opportunités d'intervention offertes par la situation « d'épouse de », se trouvant à Paris, et la revendication d'une liberté d'initiative personnelle. Suspectée d'avoir favorisé les affaires d'un proche, elle s'en défend : « Je n'ai point fait avoir de commission au parent de mon mari... Ce parent est venu à Paris, il a fait lui-même une soumission par écrit au bureau de la guerre pour deux cents chevaux de remonte. La soumission a été acceptée ; le prix des chevaux fixé par le ministre ou par ses agents, et le marché conclu comme cela se pratique entre tous les fournisseurs. Je doute que le parent de mon mari puisse bénéficier de son marché » du fait de la lenteur des

(49) La loi sur l'accaparement a été votée sur le rapport de Collot d'Herbois, le 26 juillet 1793.

(50) BM Poitiers, ms 569. 
bureaux. Elle ajoute à nouveau en note : « Ma correspondance et celle de mon mari, prouveront que je ne suis pour rien dans aucun marché ».

La fin de la brochure se retourne en dénonciation de l'accusateur, Gabriel Thibaudeau. Lui-même doit sa carrière à la mansuétude de Piorry : "Tu dois à mon mari ton entrée dans les bureaux de la Législative ». De plus, elle met en cause sa faiblesse morale, qui le disqualifie comme accusateur : « À peine entré dans ces bureaux, tu es venu habiter le même hôtel que moi ; c'est là que j'ai vu mille traits d'incivisme et d'immoralité de ta part. Ces traits d'incivisme sont ton peu d'attachement à tes devoirs, et le ridicule amer que tu venais étaler sans cesse aux yeux de mon mari sur les opérations des membres les plus patriotes des comités et de l'Assemblée en général. » L'accusation devient explicitement politique. Elle nous renseigne aussi sur les conditions de vie des députés, l'importance de l'interconnaissance associée à une forme de promiscuité, propice à des médisances pouvant se convertir en rumeur ou en délation : «Ne t'ai-je pas dit mille fois que l'Assemblée nourrissait en toi un serpent dans son sein ; et mon mari, que tu épiais nuit et jour, n'a-t-il pas été obligé parfois de s'indigner de tes propos et de te mettre hors de sa chambre? Qu'as-tu fait pour la liberté depuis que je te connais ? Jamais on ne t'a vu fréquenter les sociétés populaires, tu les as au contraire toujours calomniées ».

La fin du texte est particulièrement intéressante quant à l'engagement politique de l'épouse. Elle y revendique un choix personnel lors de la révolution du 10 août et de l'entrée en république. Elle exprime avec véhémence l'opposition entre son courage de combattante et la faiblesse morale de son accusateur : «Le 10 août, tu étais renfermé dans ta chambre, tandis que moi, placée sur le sommet de la sainte montagne, entre les balles et les boulets, je criais à pleine tête, vive la liberté et l'égalité, à bas les tyrans, à bas les traîtres !». Pour l'épouse de Piorry, il n'y a pas d'opposition ou de distinction de sexe entre elle et Gabriel Thibaudeau, mais différence de courage et d'engagement politique. Où était-elle exactement ? Elle ne le dit pas, mais elle était au cœur du combat, peut-être parmi les femmes qui ont forcé l'entrée des Tuileries. Elle poursuit, mettant en avant à nouveau la très précise connaissance de la vie de ses proches : « Tu as été j'en conviens à Chalons au mois de septembre 1792 ; mais tu n'as été que huit jours au camps ; tu t'es rendu d'abord à un hôpital, par rapport à un mal de jambe, tu es ensuite revenu à Paris, au bout d'un mois de campagne pour te reposer de ton voyage. Après ce repos tu devais retourner à ton poste, mais tu as sollicité ton congé et tu l'as obtenu. Quelles sont les compagnies que tu as fréquentées depuis deux ans ? Ce sont des aristocrates ou des femmes prostituées ». Le propos n'est pas ici de 
débattre de cette accusation de fréquenter des « aristocrates » et de savoir ce qu'elle recouvre comme imputation sociale. L'important est de relever cette posture de femme, plus âgée de quelques années seulement, à la fois vigilante et sûre d'elle-même, dénonçant la lâcheté du jeune homme face à l'engagement militaire dans un moment de grande mobilisation patriotique, ainsi que ses mauvaises fréquentations : «Quand tu as vu des aristocrates, je t'en ai fait des reproches, tu m'as répondu que tu vivais avec tout le monde et que mon mari ne prospérerait jamais parce qu'il ne soutenait pas les deux partis. Quand je t'ai reproché ton inconduite auprès des femmes prostituées, même en présence des personnes qui venaient pour me voir, tu me répondais que tu n'avais d'autres passions et d'autres moralités que le plaisir, et que tu te f... du reste ».

La femme Piorry l'accuse encore d'avoir abusé de leur proximité, de sa confiance, et de l'absence du mari pour la trahir: "Quand mon mari était en commission à Poitiers tu es venu adroitement me demander à mettre tous mes paquets à la poste ». Puis elle remet en cause l'explication fournie par Thibaudeau, mettant en avant sa situation d'épouse de député et le respect que cette place sociale impose : « Eh bien !, écrit-elle, je t'accuse d'avoir intercepté la lettre à Bernazais ; c'est toi qui a mis le cachet du comité des Contributions publiques, et si quelque membre du comité a été mis dans la confidence, il ne peut y avoir que Lebreton, député, contre révolutionnaire qui a été ton ami, ton protecteur, et qui est aujourd'hui en état d'arrestation ; comment se peut-il qu'un membre du comité t'envoie chercher, qu'il prenne sur lui de te confier la lettre de l'épouse d'un député, tandis qu'elle devait rester à la poste, ou être remise au comité de Sûreté si elle contenait des faits graves $»^{51}$. Elle renforce son argumentaire par l'évocation d'une situation similaire dans un passé proche. On retrouve la densité des relations entre ces acteurs, s'épiant, se toisant, se menaçant, en venant parfois aux mains ; une proximité physique, affective, évoquée ici de façon très directe par Adélaïde :

«Rappelle-toi que tu as soufflé une des lettres de l'épouse d'un autre député, que tu t'en es amusé... que je t'ai arraché la lettre en te menaçant de te dénoncer comme le plus perfide de tous les hommes. Tu es venu m'annoncer il y a plusieurs jours, que tu allais avoir la direction de la

(51) Lebreton est l'un des représentants de l'Ille-et-Vilaine, voir Auguste KUSCINSKI, op . cit. Il fait partie des députés d'Ille-et-Vilaine qui s'opposent à Robespierre, aux montagnards, dénoncent les pressions des clubs parisiens sur la Convention ; voir Roger DuPUY, « Aux origines du "fédéralisme" breton : le cas de Rennes (1789-mai 1793) », Annales de Bretagne et des pays de l'Ouest, 1975, volume 82 , p. 337-360. 
messagerie de Poitiers ; tu m'as prié de faire appuyer ta pétition par mon mari, et sur la demande que je te fis si la place était bonne, foutre, me dis-tu, "je sais bien que je suis pas un bougre trop calé de toutes les manières, mais j'aurai un contrôleur qui a le fil". Et te voilà aujourd'hui, malgré ton incivisme, malgré ton ignorance et ton immoralité, devenu le dépositaire des secrets des familles, de la vie et de la fortune publique, et de la circulation de tout ce qui peut emmener le succès de la révolution ».

La brochure se termine par une prise de parole du député qui répond à son tour à la question des liens entre les époux dans l'espace politique républicain. Piorry inscrit, en effet, son jugement des faits incriminés dans la perspective d'une exigence de rigueur morale propre aux républicains, en même temps que de devoirs entre époux : «Un mari républicain ne peut fermer les yeux sur les imputations faites à son épouse ; s'il la reconnaît coupable, il doit provoquer lui-même toute la sévérité nationale. Fort de ces principes, j'ai interrogé mon épouse ; j'ai scrupuleusement examiné sa conduite, et pièces en main, je suis à même de prouver toute son innocence ». Dans la fin de son exposé, il confirme que l'atteinte portée à l'épouse d'un député est bien une atteinte politique, une atteinte à la souveraineté nationale :

« Je dois donc, écrit-il, par amour pour la liberté et pour la souveraineté du peuple, dénoncer aux vrais Républicains l'individu sans principe et sans mœurs, qui osa injustement avilir le caractère le plus sacré. J'appuie à cet effet, l'adresse de mon épouse dans tout son contenu, et je la soutiendrai de toutes mes forces au comité de Sûreté générale de la Convention ».

Le texte met crûment en évidence l'enjeu politique que sont les faits et propos des «femmes de ». Il décrit la tension existant entre l'affirmation de la liberté de la citoyenne qui met des limites à ce que son statut d'épouse peut lui imposer comme contraintes, et l'existence de ces contraintes spécifiques d'épouse de député en République.

L'existence des épouses de députés, dans le moment de grand déploiement des significations politiques des faits sociaux qu'est l'entrée en république, n'est donc pas sans enjeu politique ; celui-ci concerne, de fait, tous les liens sociaux basés sur du non politique, jusqu'aux liens familiaux et amicaux. Que faire de ces proximités, de ces fidélités, de ces possibles connivences dans un monde de citoyens libres et égaux ? On connaît les limites imposées par les lois à l'exercice de fonctions politiques dans les 
mêmes structures par des hommes de la même famille ${ }^{52}$. La question est posée, qui l'est encore aujourd'hui dans les démocraties, de l'exercice de la vertu républicaine face aux contraintes des affinités, de l'interconnaissance, des dépendances économiques, face à la complexité des enracinements, notamment hérités. Elle n'est certes pas posée explicitement concernant les épouses, que la situation juridique maintient comme toutes les femmes hors des fonctions publiques. Mais la réalité des diverses pratiques sociales des épouses amène cependant à penser la forme juste qui peut combiner la légitime liberté d'action de la citoyenne et ce que ses liens intimes avec l'élu peuvent inclure de possibles privilèges, d'influences et d'autorité illégitime.

Anne JOLLET Université de Poitiers UFR CRIHAM (EA 4270) Sciences humaines et arts, 8 rue René Descartes, 86073 Poitiers cedex 9 anne.jollet@univ-poitiers.fr

(52) Voir ces limites concernant, par exemple, la composition des comités de surveillance et des municipalités dans Jacques GODECHOT, op. cit. 